\title{
Ousselat (Jebel — ; Tunisie) : Préhistoire et art rupestre
}

Sophie Yahia-Acheche

\section{OpenEdition}

1 Journals

Édition électronique

URL : https://journals.openedition.org/encyclopedieberbere/2870

DOI : 10.4000/encyclopedieberbere.2870

ISSN : 2262-7197

Éditeur

Peeters Publishers

\section{Édition imprimée}

Date de publication : 2 juin 2013

Pagination : 5986-5993

ISBN : 978-2-7584-0194-0

ISSN : 1015-7344

\section{Référence électronique}

Sophie Yahia-Acheche, "Ousselat (Jebel - ; Tunisie) : Préhistoire et art rupestre », Encyclopédie berbère [En ligne], 36 | 2013, document 055, mis en ligne le 12 mars 2021, consulté le 17 février 2022. URL : http://journals.openedition.org/encyclopedieberbere/2870 ; DOI : https://doi.org/10.4000/ encyclopedieberbere.2870

Ce document a été généré automatiquement le 17 février 2022.

(c) Tous droits réservés 


\title{
Ousselat (Jebel — ; Tunisie) : Préhistoire et art rupestre
}

\author{
Sophie Yahia-Acheche
}

1 Le Jebel Ousselat [Fig. 1] est une petite montagne de Tunisie centrale (Région de Kairouan), d'une superficie de $135 \mathrm{~km}^{2}$ et une altitude peu élevée de $895 \mathrm{~m}$ au Jebel Chaïeb, son point culminant. Il s'agit d'une formation géologique d'âge Crétacé et Éocène inférieur, constituée de calcaires durs et d'une alternance Marno-calcaire. Le Jebel Ousselat est massif, les sommets sont dépourvus de végétation car ces formations calcaires trop épaisses, sont très perméables. Le paysage y est aussi très contrasté selon l'orientation des versants et l'altitude qui font varier la pluviométrie. Il se trouve dans une aire sèche malgré sa position géographique et bénéficie d'un climat semi-aride. 


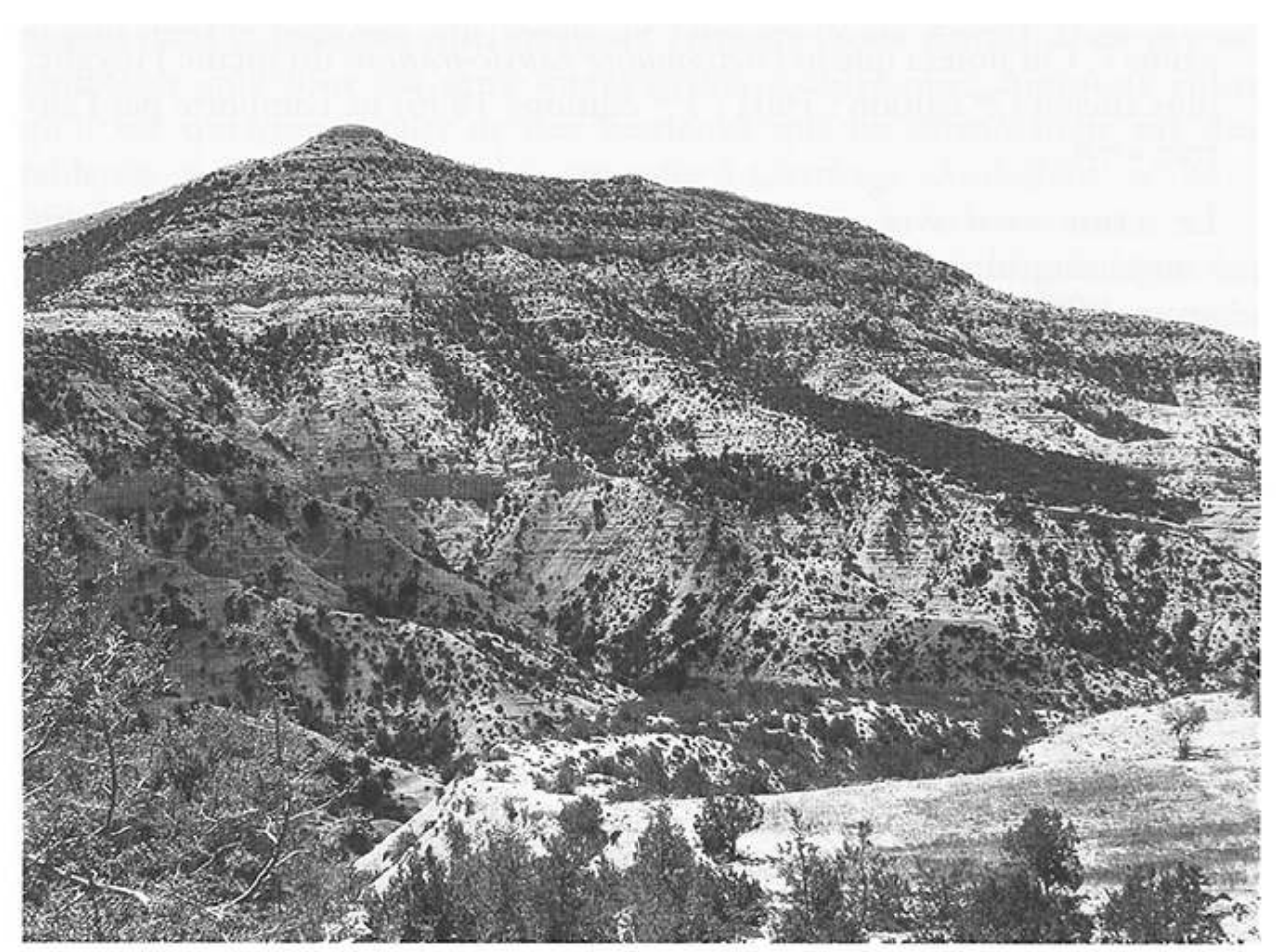

Fig. 1 : vUE générale de LA VALLÉE de L'OUed ELLOUZ, J. OUSSELAT (s. yahia)

Le Jebel Ousselat qui, avec le Jebel Bouddabous va se raccorder au centre de la Dorsale tunisienne, fait partie d'un alignement montagneux appelé axe Nord-Sud aux altitudes modérées (moins de 1000m). Nous sommes dans l'Atlas tunisien, au Nord de la plateforme saharienne, dans une zone qui était occupée en partie par l'île de Kairouan qui existait au Crétacé inférieur. Du haut de cette montagne, on domine les plaines de Kairouan, d'Ousselatia et la dépression de Haffouz. Une dépression de 220 à $400 \mathrm{~m}$ d'altitude seulement au sein du Jebel Ousselat en a fait une zone continentale de passage lors des migrations humaines entre le Sud et le Nord. Les cavités calcaires creusées par la disparition des marnes ont offert des abris aux hommes. Le Jebel Ousselat abrite des vestiges archéologiques de toutes les époques, son passé préhistorique n'a été que récemment mis au jour.

3 L'occupation humaine est très ancienne et pérenne dans la région d'Ousselatia, les montagnes et les plaines environnantes. Les plus anciens gisements sont acheuléens dans la vallée de l'oued Merguellil (Gragueb \& Karray 1991). Au Jebel Ousselat les prospections préhistoriques sont relativement récentes. Les peintures rupestres ont été découvertes par Alix Martin en 1988 (Gragueb et al. 1989). Les recherches archéologiques systématiques n'ont débuté qu'en 2001 (Ben Nasr 2001, 2007 ; Yahia 2007, 2009).

4 L'étude du matériel lithique de surface indique une prédominance des cultures épipaléolithiques voire néolithiques (Ben Nasr 2007, p. 204). Le matériel lithique en calcaire et silex présente une majorité d'éclats. Le calcaire est certainement la principale ressource utilisée par les Préhistoriques pour la confection de leur outils. Il est présent sous forme de rognons de différentes tailles. Des affleurements de silex de différentes couleurs (gris foncé et clair, brun, etc.) sont signalés dans les jebels du Nord de la Dorsale (carte géologique 1/50 000 e 3, Aïn Jeloula, 1998, 2000) et dans le Jebel. Ousselat (Ben Nasr 2007). Le silex est également présent en formation secondaire sur 
les pentes des massifs et dans les lits des oueds. Les Néolithiques ont également rapporté du silex allochtone, probablement recherché pour ses qualités techniques supérieures.

5 Les habitats holocènes sont des rammadiyat de plus ou moins grande envergure, tellement lessivées parfois qu'elles sont à peine repérables. L'érosion est très importante et ces vestiges fragiles finissent souvent dans les pentes raides du massif ou au fond des oueds. Il s'agit généralement d'un habitat en plein air que l'on retrouve aussi, au Jebel Ousselat, en contrebas de grottes (Damous el-Ghoula - Oued Majel 2), d'abris sous-roche (Ghorfat Rmada) ou dans de petites cavités (Kef Skhira). Le versant Nord du Jebel, bénéficiant de pentes plus douces, a sans doute été plus favorable aux installations humaines.

6 Le Jebel Ousselat abrite aussi l'ensemble rupestre le plus riche connu à ce jour en Tunisie, avec une vingtaine d'abris sous-roche et autres cavités ornées (Ben Nasr 2007 ; Yahia 2007, 2009). La concentration des stations rupestres permet de nous pencher sur les critères qui ont pu orienter le choix des artistes : Les artistes ont orné de grands abris sous roche aussi bien que de toutes petites cavités, avec une préférence pour les abris de dimensions moyennes. Les stations rupestres se concentrent surtout sur le flanc sud de la montagne, à la jonction de plusieurs oueds (Melg-el-Ouidien). Plus généralement ils suivent le réseau hydrographique ou les sources. L'étude comparative que nous avons menée, laisse apparaître que toutes les stations n'ont pas eu la même importance aux yeux des hommes. Nous distinguons deux catégories de stations rupestres. La première catégorie concerne des stations de taille et d'importance variables qui ont été investies par un seul groupe. Elles ont pu constituer une courte étape dans le parcours des populations préhistoriques ou protohistoriques. Les secondes, en revanche, semblent revêtir une importance toute particulière car des hommes de cultures et d'époques différentes les ont occupées et sont venus graver et peindre sur la pierre leur imaginaire, sans jamais interférer sur les représentations de ceux qui les ont précédés. Véritables "Saint des saints", deux abris sous roche se distinguent au Jebel Ousselat par une taille conséquente, une situation privilégiée près d'une source - et de longues et lisses parois, merveilleux supports pour des artistes prolifiques.

7 C'est le cas par exemple de l'une des trois stations rupestres de l'Aïn Khanfous [Fig. 1bis] (JO/AK03) à laquelle on accède en remontant le cours de l'oued Bouddabous, près du lieu-dit Dar el-Bey. Les panneaux ornés comprennent 243 figures, de styles et d'époques différents. L'abri de l'Ain Khanfous n'est pas facilement repérable sur le flanc abrupt, son accès est périlleux et on y pénètre d'un seul côté, le gauche. Ainsi, il y a un sens obligatoire à la visite et probablement à la lecture des scènes représentées, les personnages comme les animaux semblent tous se diriger vers le fond de l'abri.

Une seule paroi longue de plus de $28 \mathrm{~m}$, extrêmement incurvée donne l'impression de pénétrer dans une cavité. Le nombre de figures augmente au fur et à mesure que l'on avance et les scènes ou groupes de figures associées se succèdent jusqu'à l'extrémité droite de l'abri. Au ras du sol, sur un pan incliné à $140^{\circ}$, se trouvent les gravures piquetées d'époque protohistorique, dans un premier registre. La présence simultanée des lances ou javelots et du cheval, et la convention stylistique des " pattes à roulettes " sont caractéristiques de l'époque libyco-berbère. Ces gravures sont pourtant associées à des inscriptions mortuaires islamiques, probablement à caractère votif (probable remploi de gravures plus anciennes). Puis, la paroi se redresse à la verticale et les 
gravures néolithiques à contour poli sont placées dans un second registre, à peu près $0,50 \mathrm{~m}$ du sol. Dans un troisième registre (de 0,80 m jusqu'à 1,80 m environ de hauteur), se trouvent la plupart des peintures. Le dernier registre supérieur qui est à plus de 2 mètres de hauteur, est occupé par la fameuse «scène des archers ", de style caballin, une scène à caractère symbolique associant plusieurs archers dont certains portent une queue postiche et un boviné.

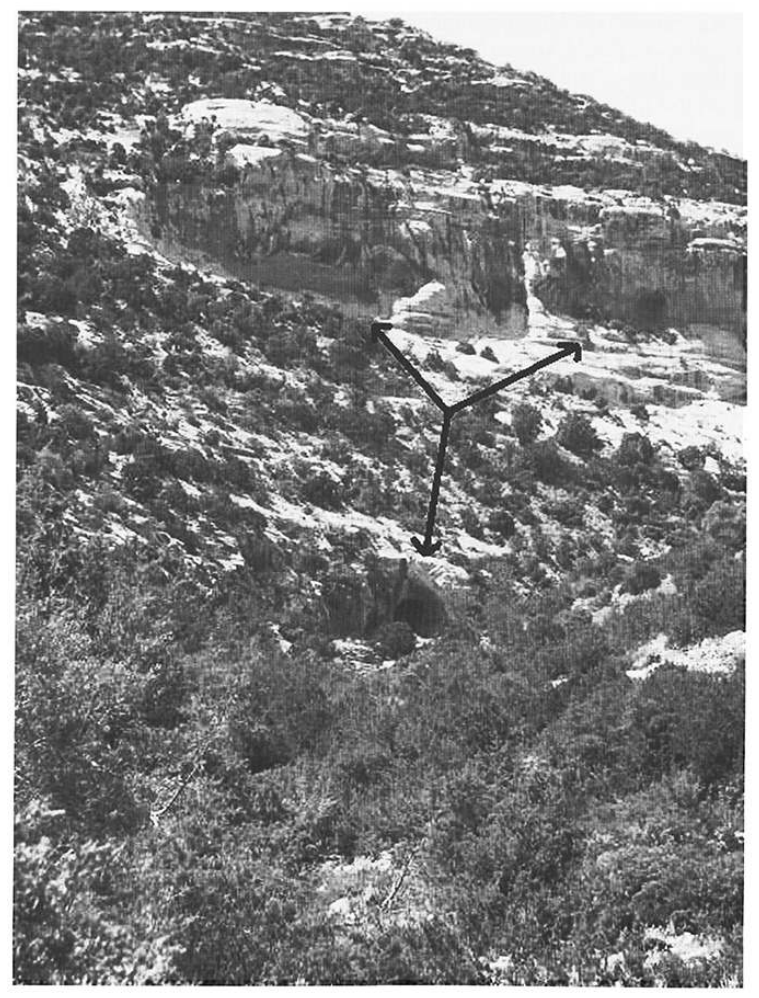

Fig. 1BIS : VUE gÉnÉRALE des ABRIS de L'AïN KHANFOUS (S. YAHIA)

9 La gravure est une technique extrêmement rare dans l'art rupestre de Tunisie. Au Jebel Ousselat, la station Ghorfat er-Rmada lui est dédiée entièrement (Yahia 2007). Elle se trouve à $715 \mathrm{~m}$ d'altitude, sur la rive droite de l'oued Ellouz. Le bestiaire y est majoritaire, composé de la grande faune sauvage et autres mammifères équatoriaux. Sur un panneau par exemple se trouvent parmi les plus anciennes gravures connues en Tunisie, que l'on peut associer au style «bubalin naturaliste ». Elles représentent des espèces de la steppe, des animaux de la grande faune qui ont pu se maintenir dans ces contrées jusqu'au Néolithique, comme le rhinocéros, le Boselaphus ou l'antilope Addax [Fig. 2].

10 Sur un autre panneau, des animaux de la faune sauvage côtoient des espèces domestiquées [Fig. 3]; des personnages et des théranthropes (mi-hommes, mianimaux) sont aussi représentés en étroite relation avec les animaux. Les figures s'imbriquent les unes aux autres. Le bâton que portent tous les personnages pourrait être le symbole d'un mode de vie pastorale, de même que l'entrave qui est placée au cou de certains bovidés. D'un style naturaliste stylisé, les espèces sont parfaitement identifiables, elles présentent de nombreux détails anatomiques mais ont la particularité d'avoir les membres exagérément allongés et terminés en pointe. Les pattes de certaines figures animales, semblent non finies. Ce mode de représentation est considéré comme l'un des critères discriminants qui les apparentent aux oeuvres de 
l'école de Tazina telle que l'a définie Muzzolini (Muzzolini 1996), même si nous sommes loin de la zone d'expansion géographique de celle-ci. De même, avec la présence du Syncerus caffer antiquus, qui s'est maintenu en Afrique du Nord jusqu'au Néolithique, nous pensons pouvoir classer ce type de gravures parmi les gravures anciennes appartenant au Néolithique. Le troisième panneau est composé d'au moins dix gravures obtenues par une succession d'incisions fines, définissant, dans leur majorité, des guerriers et leur monture. Certaines sont à peine lisibles par un enchevêtrement inextricable de lignes et d'incisions. D'autres apparaissent clairement: des cavaliers debout sur leur monture ou encore les personnages qui se tiennent par la main. Ces gravures sont attribuées aux époques récentes (protohistoriques et historiques). Elles sont reconnaissables par la technique du piquetage et une extrême simplification des graphismes.

Le Jebel Ousselat n'est pas la seule région en Tunisie où des figurations rupestres ont été repérées. Longtemps absente des cartes de répartition de l'art rupestre nordafricain, la Tunisie comporte à ce jour deux autres ensembles ${ }^{1}$ rupestres, Zaghouan (Jebibina, au Nord), et Ghomrassen (Sud-Est) ainsi que quelques stations isolées qui couvrent une large bande du sud-ouest au sud-est de la Tunisie (Tamerza, Gafsa, Redeyef, Sidi Bouzid).

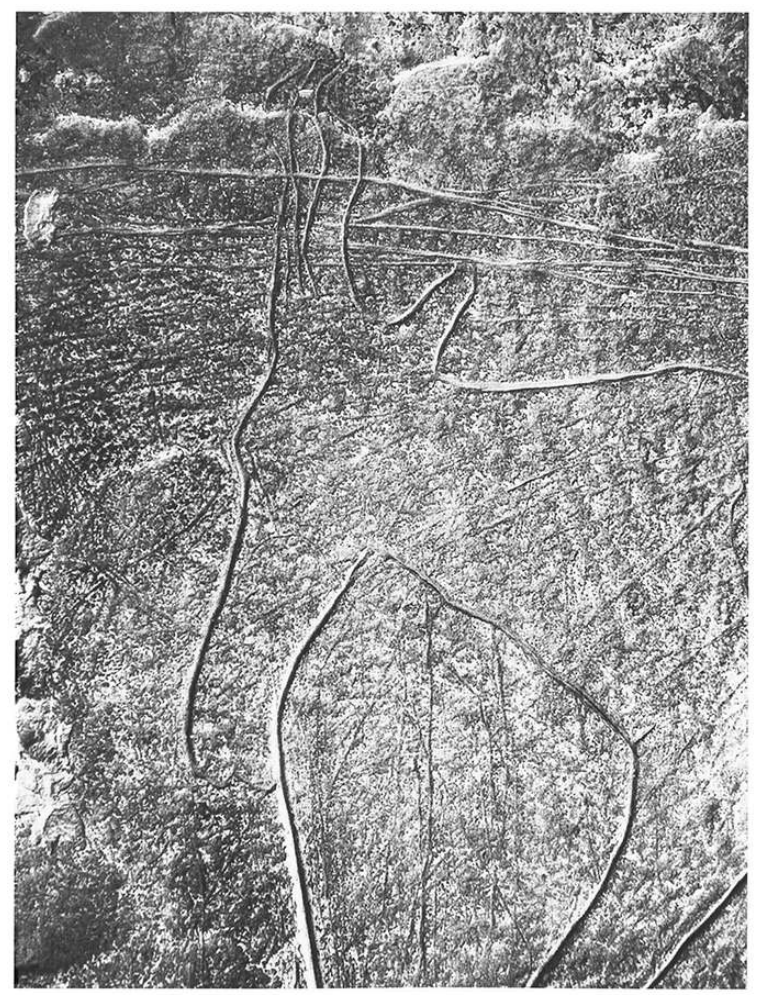

FIg. 2 : GRAVURE DE STYLE " BUBALIN NATURALISTE ". GHORFAT RMADA. J. OUSSELAT (S. YAHIA).

Le corpus rupestre de Tunisie, en l'état actuel des recherches, commence à livrer des données chrono-culturelles intéressantes. Les plus anciennes représentations de Tunisie sont des gravures à contour poli de patine totale ou foncée qui représentent des traits subparallèles dits « capsiens » ainsi que des animaux d'une faune sauvage témoin des épisodes humides intervenus avant le dernier grand « Aride post néolithique » (le rhinocéros, le grand buffle antique, le lion, l'antilope,...). Celles-ci sont présentes au Jebel Ousselat, elles sont rares et éparses dans le Sud (Gafsa, Sidi Bouzid). Quant aux 
peintures, rien ne permet d'affirmer qu'elles puissent dater de l'Épipaléolithique ou du Néolithique ancien. La période bovidienne est peu attestée (Ousselat, Jebibina). En revanche, la période du cheval qui marque le passage de la fin du Néolithique à la protohistoire, est relativement bien représentée dans les peintures (Ousselat, Tamerza, Ghomrassen). Enfin pour les périodes camélines, le Jebel Ousselat livre tous les exemples connus. Les inscriptions épigraphiques arabes nous mènent jusqu'à une période historique relativement récente dans les abris de l'Aïn Khanfous ou à Zaghouan (Nord).

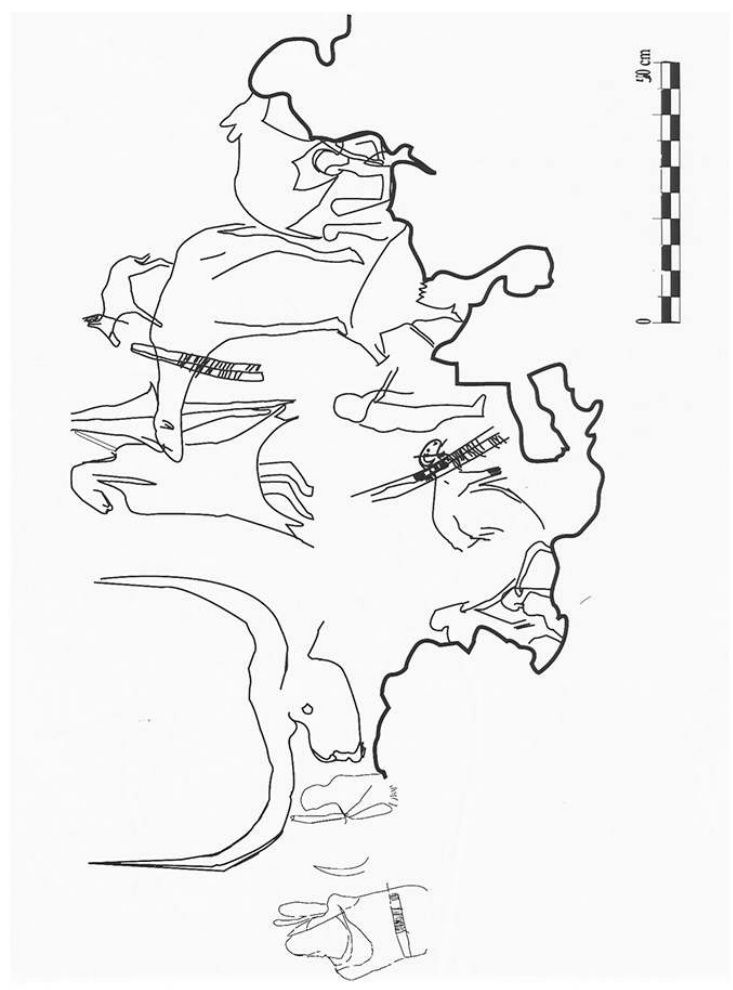

FIg. 3 : RELEVÉ DE gRAVURES. NÉOLITHIQUE RÉCENT. GHORFAT RMADA. J. OUSSELAT (S. YAHIA).

La prospection du Jebel Ousselat a montré la richesse et la diversité des vestiges archéologiques, ainsi que l'importance et l'ancienneté de son art au sein du corpus rupestre de Tunisie. Par sa situation géographique et ses correspondances stylistiques, l'art rupestre de la région s'intègre dans l'ensemble Atlasique et plus particulièrement dans l'Atlas tellien algérien (Sud de la région de Constantine et région de Tebessa). Aussi, de grandes similitudes ont été mises en évidence dans les gravures comme dans les peintures. Des éléments de comparaison pourraient nous faire envisager une identité culturelle entre les deux régions. S'il y avait eu des traditions iconographiques communes, il serait intéressant de trouver les liens qui unissent les populations et les voies de circulation ayant permis les contacts, dans une région extrêmement vaste et escarpée. 


\section{BIBLIOGRAPHIE}

BEN NASR J., 2001 - « Quatre abris peints découverts au Jebel Ousselat », Préhistoire Anthropologie Méditerranéenne, t. 10, p. 1-7.

BEN NASR J., 2007 - Recherches sur le peuplement préhistorique du Jebel Ousselat (Tunisie centrale), Thèse de Doctorat, Université de Provence, Aix-en-Provence, 2 vol.

GRAGUEB A., HARBI-RIAHI M. ; M'TIMET A. ; zOUGHLAMI J., 1989 - « Nouvelles découvertes des représentations rupestres de Tunisie. Jb. Ousselat (Tunisie Centrale) », Bulletin des Travaux de l'INAA, fasc. 4, p. 41-64

GRAGUEB A. KARRAY R., 1991 - « Découvertes du Paléolithique inférieur en Tunisie centrale (note préliminaire) », Bulletin des travaux de l'INAA, IV : 64 et s.

MUZzolini A., 1996 - Les images rupestres du Sahara, édition de l'auteur, Toulouse, 447 p.

YAHIA-ACHECHE S., 2007 - « Les gravures de Ghorfat-er-Rmāda (Tunisie centrale) », Cahiers de l'AARS, 11, p. 145-156.

YAHIA-ACHECHE S, 2009 - L'art rupestre de Tunisie. Inventaire et Analyse. Ses relations avec les foyers artistiques de l'Algérie et la Libye voisines, Thèse de Doctorat, Paris 1-Panthéon-Sorbonne, $412 \mathrm{p}$.

\section{NOTES}

1. Les ensembles sont définis par plusieurs stations rupestres présentes dans une même zone géographique. Ils ne présentent pas forcément une unité chronologique.

\section{INDEX}

Mots-clés : Art rupestre, Capsien, Néolithique, Néolithisation, Préhisoire, Tunisie 